\title{
GROCIO-PUFENDORF ANTE MAYÁNS-CAMPOMANES
}

\author{
Alfredo MARTÍNEZ ALBIACH
}

Prémbulo: ¿Grocio o Pufentorf?

Al introducirnos en la centuria dieciochista, en 1963 bajo la orientación de José Luis López Aranguren', ambicionamos comprender la realidad histórico-social de la España actual. Ésta «no es inteligible sin verla originarse (...) desde mediados del siglo XVIIL².

Hoy, tras un largo recorrido «hacia atrás y adelantew" volvemos a plantcarnos un interrogante`: ¿quién influyó más Crocio (1583-1645) o Pufendorf (1632-1694) en nuestros ilustrados dieciochistas?

Así pues, sin afán de paralelismos, y en línea con Antonio Mestre Sanchís". contemplamos nuestra centuria decimoctava con dos personajes que cronoló-

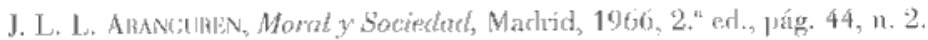

J. Marias, Le Espoña posible en fienesos de Centos Hh, Marlrid, 1966, pág. 233.

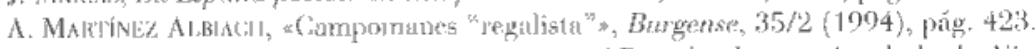

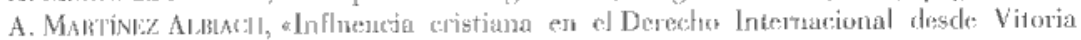
"Grocios, Burgense, 32/2 (1991), púg. 343.

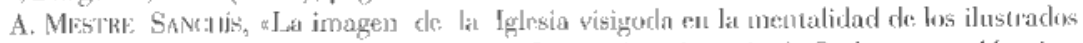
espanoles. El caso de Mayáns y Cimpomanes", en Homenaje at Antonio de Bethencourt Massien, Snuta Cruz de Tenerife, Sentinario de Fumarialates, 1906, jág. 483. 
gicamente la cubrem, y que dicho autor los une en un aspecto: Gregorio Mayáns y Siscar (1699-1781) y Pedro Rodríguez de Campomanes y Pérez (17231802): ael análisis de crítica histórica venía a confirmar las regalías de los visigodos, ideal de la iglesia nacional, que deseaban los regalistas ilustrados españolesn.

Si bien el contacto personal de Mayáns y Campomanes se produjo en 1766 (iniciándose una profunda correspondencia evistolar hasta el fallecimiento rlel crudito valenciano), su relación ideológica surgió con anterioridad", tanto en el referido ideal para los regalistas ilustrados hispanos como con la apertura a textos exiranjeros (incluidos los protestantes Grocio y Pufendorf), pues según Mayáns en conrespondencia con el duque de Fluéscar (24 de diciembre 1746)7. un caloblico tiene que saber las herejías que har unido los iusnaturalistas a sus teorías políticas.

\section{Relacion ideológica rntre Meyuns y Campenturnes}

Gomn ya hemos esudiado el catolicismo y eregalismo» de Ciampomanes". ahora trataremos de comsiderar los mismos raracteres de Mayáms, gracias a Mesire?.

Nacido Gregorio Mayáns en Oliva (Valencia), en cuya parroquia de Santa María fue baulizado, Ia vinculación política de su padre con el Archiduquo Garlos en la Guerra de Sucesión española provocó disgustos y consecuencias negativas de largo alcarte. I éa lamilia luvo que abandonar Valencia después

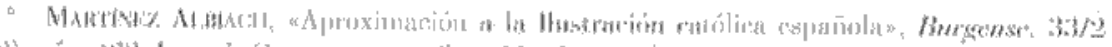

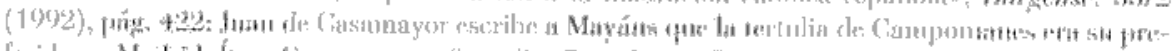

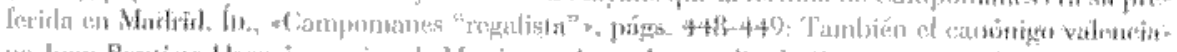

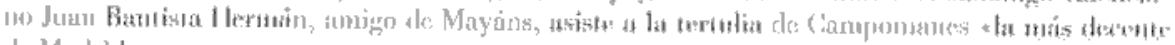
let Madist.

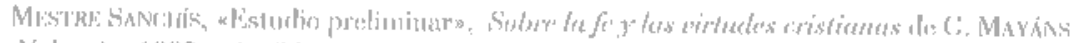

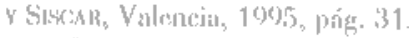

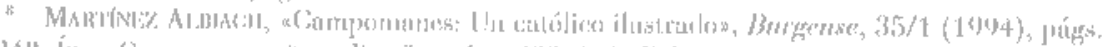

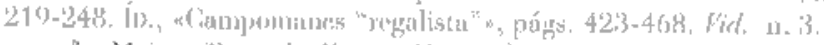

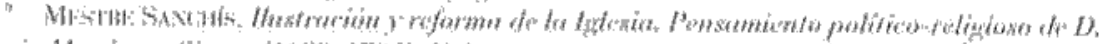

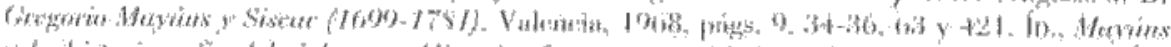

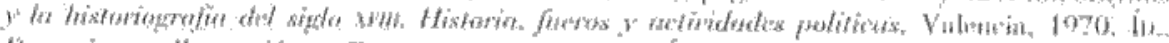

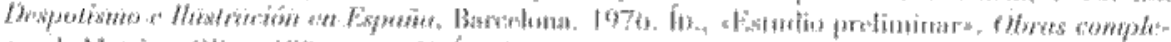

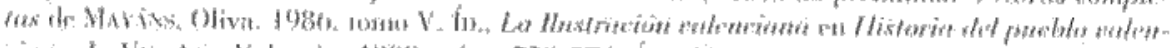

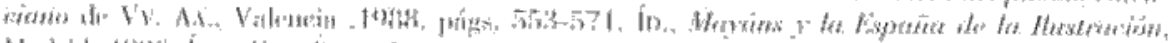

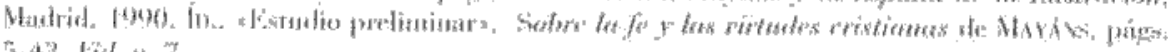
$5-4.3 .1 \mathrm{Hl}, \mathrm{H}, \mathrm{T}$. 
de la batalla de Almansa (1707) y trasladó su residencia a Barcelona. La relación de los Mayáns con la corte austríaca debió ser ímima, pues el Archiduque concedió la gracia del bugar y encomienda de Museros (Valencia) al padre, mientras agraciaba al joven Cregorio con el hábito de Santiago. Estas circunstancias explican que Mayáns estudiase latín en el colegio barcelonés de Cordelles regido por los jesuitas (Gramática, Retórica y Poética latinas), y a los quince años recibiera la clerical tonsura (viernes 21 de diciembre 1714) para dedicarse a la enseñanza. Pese a las críticas posteriores del erudito a la docencia jesuítica, sus conocimientos de latín demuestran una espléndida formación humanistica.

Finalizarla la Cuerra de Sucesión, la familia Mayáns (en julio de 1713) regresó a Oliva, y el joven Gregorio, tras leer libros de Jurisprudencia bajo el influjo de su abuclo materno, pudo iniciar sus estudios de filosofía en la miversidad de Valencia después de aquella Navidad de 1713 hasta marzo de 1716, centrándose luego en el Derecho.

Resulta muy comprensible que, con la educación en el colegio de los jesuitas, se adscribjese a la escuela antitomista. Pero al humanista, educado en la lectura de los clásicos, le resultalua molesta la jerga escolástica y estudió Filosofía, en palabras del đleán Martí, «el arte exercendi praceonium», o como traduce Luis Gi], «hablar a gritos como los pregoneros», sin dejar los estudios clásicos. Esle humanismo le permitió ser conocido en el mundo inclectual valenciano: mientras lo encargaron unos epigramas en forma de laberinto para commemorar la muerte de la reina María Luisa de Saboya (1714) que, lédos al revés, conservan su sentido, su fama le permitió acceder a unas cordiales relaciones personales y directas con los representantes más caracterizatos de los novatores valencianos. Así se comprenden la amistad con Tosca, corachán a Ínigo, anmque Mayáns decidió esudiar Derecho a partir de 1710 , como ya indicamos.

Esa dualidad explica los primeros pasos en la vida intelectual de Mayáns. No era Jurispmudencia una facultad especialmente bien dotada en Valencia, y menos después de unos años de abandono académico durante la Guerra de Sucesión. Estudió por su cuenta las Instituciones de Anold Vinnen con la colaboración de Juan Bautista lerrer, catedrático de la Lniversidad y años después obispo de Jugo. En consecueneia, la fanilia Mayáns hizo un esfuerzo económico para que el joven y brillante estudiante completara su formación jurídica en la lamosa universidad de Salamanca, en octubre de 1719.

En contraste, los esturlios nédicos y matemáticos (evidente influjo de los novatores) aleanzahan en Valencia un momento de esplendor. Lo comu- 
nicaba (13 mayo 1720) a Mayáns, ya residente su Sulananca, el P. Jerónimo Juliám, prepósito de la Conpañía en Valencia e intimo de la familia: «Si no luera por no aumentar portes, te hubiera pnviadv conclusiones de Medicina y vieras to que yo no acabo de admirar rque, haluiendo estado la Universidad 15 años sin orden, ni maestros, no ubstante con la noble aplicación de los ingenios, unos a enseñar sin interés y otros a aprender con sólo saber, no sólo no han descapcido las ciencias, sino que con asombro se han adelantados. El texto demuestra, jor supuesto, que los novatores desturolienom su actividad cienríli-

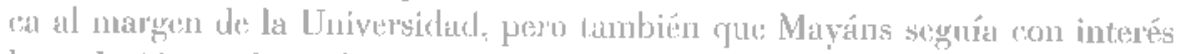
la evolueion juleletollal valenciana.

La pluralidad de intereses culurales constinyó uno de los caracteres de la personglidarl intelectua] de Mayúns, pues no olvidaba sus estudios de Deresho, ya mencionados. Influido por los comsejos a su cirarlo abuelo materno (lwan Antonio Sistar), que halóa sino alunumo en Salamanca durante Ins

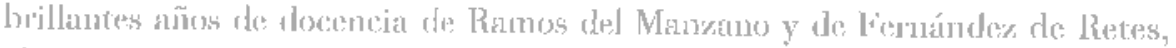

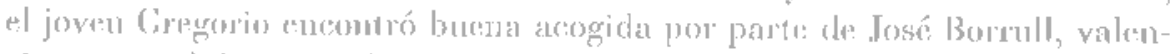

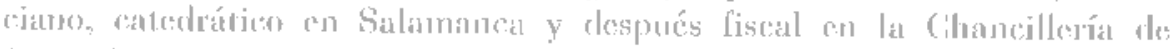

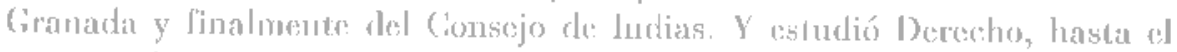
extreme de que dompró manuscritos de los grander juristas salmanticensest

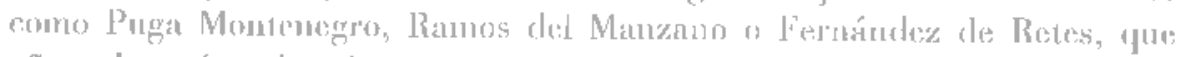

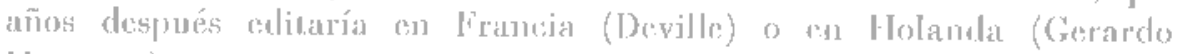
Mermation).

Sin embargo, su dedicueión al cultivo de les clásieos ena por todos conoci-

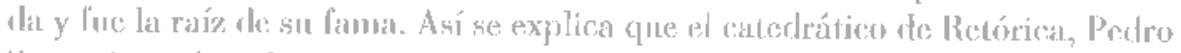

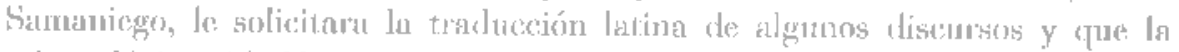

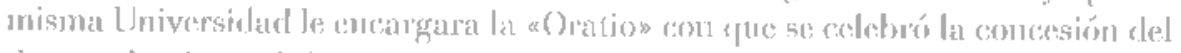
doctorado al ratedrático José Borrull. Fn este sentido, no teja de sorpmender que se le instara, apsenas Ilegado a Salamanca, para que aceptase el restorado do la Universiclad. Las palabras con que narra semejante ofreciniento a su

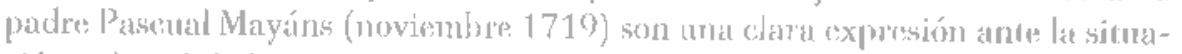
uión cultural de la Unjversidart:

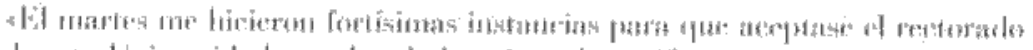

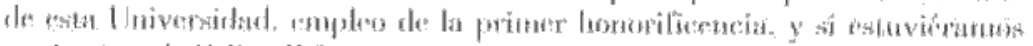

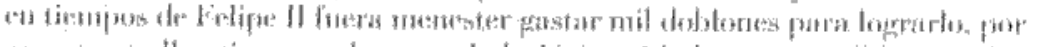

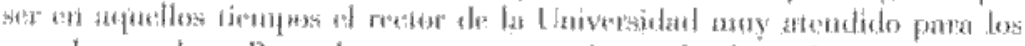

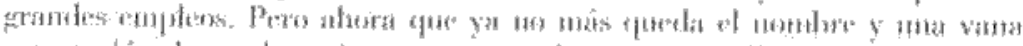

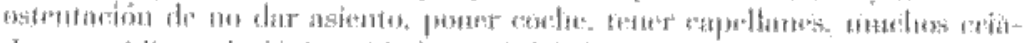

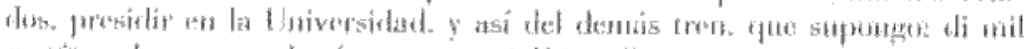

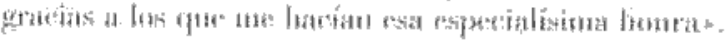


Decartencia cultural de la Universidad, pero también reconocimiento de la lama del joven Mayáns. Fanea que, de nuevo en la límea del humanismo, ammentaría con rapidez. El 2 de diciembre de 1720 escribía una car1a latina al citado Manucl Marí, deán de Alicante, con quien establecería una interesantísima relación epistolas. Martí (1663-1737) había estudiado latín, lilosolía y teología en Valencia. Hastiado de la escolústica y ávido de completar su Pormación humanista (latín y griego) inarehó a Roma donde alcanzó merecida fama por la participación en acatemias (Arcades, Infecturdi) y la colaboración en los trabajos históricos del cardenal Sáenz de Aguirre (Collectio maxima conrilionum Hisprniue (1693-1694)) con la edición de los concilios visigodos; ponía al alcance de los estudiosos las fuentes documentales esenciales, calificadas por Camponanes ucomo el manaurial más puro de derecho canónico de la iglesia española y en que se apoyan los fundamentos del Patronato universat» ${ }^{10}$.

$Y$ es que el propio Camponanes fue comisionado en 1751 por la Academia de la Flistoria a la Biblioteca de El Escorial para investigar sobre los concilios hispanos. Por lal trabajo el asturianos será nombrado académico supernumerario (10 septiembre 1751)": no olvidemos al aceeso de Campomanes a la citata Academia de la Historia (5 abril 1748) después de publicar (un año antes) sus Discrtaciones históricas de la Orden de Caballería de los Templarios; lampoeo su raduceion con Migned Casiri de una parte del códice árabe de Abu Zacaría, y olros trabajos urgalistats y econónicos. De ahí que clos lusstoriadores de Cinmpomaues (un jurista, Coronas, y 110 economista, Llonbart) hablan del avicjo ideal de la iglesia nacional gótica», especialmente vivo a pratir del Concordato español con la Santa Sede en 1753, si bien ese ideal es anterior a dicho Concordato'? inchuso a la llegada de Campomanes a Madrid on 1742 bajo la direceción de «los dos oráculos» (Juan José Ortíz de Amaya, y Miguel (ajer), y a la estancia de Mayáus en la Corte como biblintecario real en 1733.

Fueron, pues, años intensos para el valenciano, y su correspondencia nos revela los problemas que acompañaron su resirlencia madrileña. Problemas políricos, en primer lugar con el Secretario de Estado José Patiño. Mayáns deseabra d cargo de cronista do Indias, vacante descle la muerte de Lnis

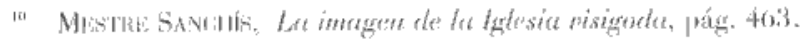

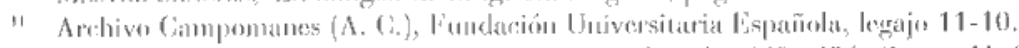

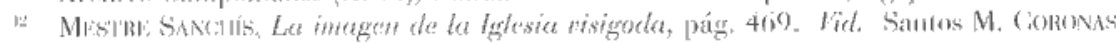

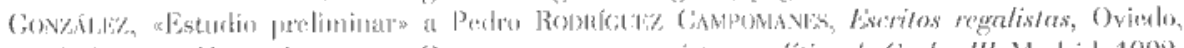

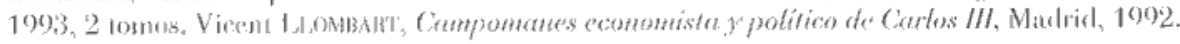


Salazar. En csa línea expuso en una conocida carta a Patiño los proyectos literarios que deseaba llevar a caho y que forman uno de los más anbiciosos y soherentes planes de reforma eultural del momento: estudios de granática y retorica, lilosofía y lógica, derecho e historia. Sobresale pl plan de ediciones de fuentes históricas, civiles y eclesiásticas, dentro de la necesidad del método crítico que se vería reformado con la publicación de los autores más significativos (Antonio Agustín, Mondéjar). Pero Patiño manifestó el desprecio más alssoluto. No sólo ignoró las peticiones del confesor real (Cuillermo Clarke) y del Secreturio de Cracia y Justicia (ularqués do la Compuesta), sino que hizo merrer la voz de que Mayáns no había treducido al latín los papeles de toma de posesión del príncipe de Pionbino. Lus ducumentos demuestrau la malicia del rumor, pues Mayáns tradujo el texto y lo entıegó al Secretario de Estado en 24 horas. Ija razón habría gue buscarla en otré parte: los anteredentes austracisles del valenciano. Én este sentido, además del favor del cardenal cienliegos, su amistad con a mobajador inglés Berjamin Keene y sus relaciones con los intelcctuales portngueses, acalbaron de envenenar el ambiente que rodeaba al vilenciano.

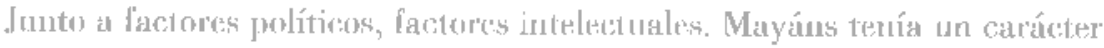
dilicil y sus relaciones con los compañeros de lá real hiblioteca no eran cordia-

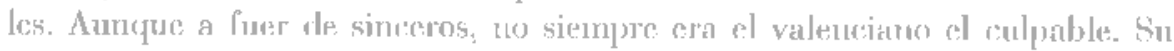
amistad con el marqués de Villena, que manifestó desde el primer momento el deseo de que Mayáns ingresara cn la Real Acardomia de lic Lengua, suscitó envidias. Lá emulación con otro bibliotecario real. Juan de Lriarie, Inen latinjsta, créo tensiones y suspicacias. Los ataques de Mayáns a los editores del (uijole de Avellaneda, autor despreciado por el propio Mayáns, en su Firla de Migael de Ceromes (1737), le yanaron la animosidad del bibliotecario nayor dol rey Blas Aubnio Nasare y del dircctor de la Academia de la Fistoria Agustim Montiano, personajes de gran peso en la política culural de la Corte.

Tuelos esos ingredicntes explotaron con la aparienón del Diurio de los literelos de España, obra de Martínez Salalrancil, Leopoldo Puig y Fraucisco [Tuerta y Vega. Da la improsión de que Nasare jugó un papel decisivo en sul gémosio y buscó la colaloración ded valenciano en aspoctos dè crítica. La negativa de Mayáns lue rotureda. Los méritos del Diorie son innegables y no vamos a analizar su inportancia y el alcance cultural de la revista. Pero dambién resulta evidente el caráter provocador de lot antores y la especial animosidad menifustala con los origenes de la lengete espetñola de Malyáms (1737). Quizás convenga resaltar en este montento, además de las diferencius centre los diaristas y Mayáns, partidarios los primeros de Fejoo que por esas fechas habían manifesiarlo ya sus 
divergencias con el valenciano, la traición de Nasarte y el carácter político adquirido por la polémira.

Las relaciones de Mayáns con Sehömberg y Mencke dieron lugar a la redaecón por pare del valenciano de Nove literaria ex Hispania, aparecida en Ac/u eruditorum de 17:31. Iá reseña era probablemente la primexa noticia literaria solore libros españoles conocida en Alemania y abaraba aspectos muy complejos: poesía, críica, listoria. Y, como cra lógico, revelaba las preferencias. $A$ esas alturas no puede sorpremdernos la censura de la obra de Fejoo, o la actsación de lentioud a la Real Acadenia en la reclaceión del Diccionario, perbonalidard de relieve e instimución emblemálica. El asunto hubiera pasado inadverdido si Mayáms no hubiese revelado en plan confirlencial su autoría a Nasare y éste communiara la moticia y el rexto a Martúnez. Salafranca. El diarista ineluyó íntegro el artículo en la revista, al tiempo que: acousaba al valenciano, eon mayor o menor claridad, de andiespanol, por haber publicado esas crítios en el extranjero eomtribuyendo a confirmar el criterio de nuestra decadencia culdural. Fra la respuesia a unas palaloras que Mayáms Labía escribo en la Vide de Cervantes: "Tabía entonces en España la ridionda costumbre de prevenir el ánimo de los lecores con muchas alabanzas, la mayor parte de ollas fabricadas por sus mismos aubores; como sucede loy en los que dan mushas juntas literarias, que profesan la crílica con poca seriedad, fiandose demasiadancate de juicios ajeuos, tal vez ignorantes y lan apasionarloss.

Esas folémicas tuvieron notables cousccuencias. En primer lugar, la acu sación de antespañol pesó solme el valenciano de manera permanente y. ex cualquier circomstancia molesta o de divergencia politico-caltural, caía sobre él la sospecha de antiespañol que servia para retirarle m favor o ammentar su marginación (carclenal Molina, (iarvajal o Rávago). Adenás, la animosidad contra los diaristas dió ocasión para que Mayáns reslactara una durísima censura contra la España primitiva de lluerla y Vega, demostrando que se basaha en un falso cronicón. La solicitud de las Reales Academias de la leengua y de la Ilistoria, que habían avalado la obra, no hizo modificar el criterio de Mayáns y al Cionsejo de Castilla permitió la publicación de la olra de Huerta y Vega. Fiuc la gota que colmó la paciencia de Mayáns y abauclonó la Corto para retirarse a ()liva ('n 1739 .

El retiro a stu casa solariega no disminuyó un ápice su actividad intelectual. Dentro de la penísula no consiguió en ma primcra etapa, que se exticnde liasta 1707. los frutos que espreraba. Fundó la Acarlemia Valenciana para lomentar los estudios de crítica histórica, pero ka persecución promovida por el 
cardenal Molina con motivo de la publicación de Censura de histortas fabulosas de Nicolás Antonio (1742), paralizó la actividad de la institución. Sólo logró publicar las Obras rronológicns (1744) y las Aduertencias a La Historia del P. Mariana (1746), ambas del marcqués de Mondéjar, Estas dificultades coinciden con la incomprensión de los políticos de Madrid: Molina, Carvajal y Rávago. Solamente Ensenada se acordó del jurista: que había colaborado infructuosamente en las polémicas regalistas cor el último equipo de Felipe $\mathrm{V}$ (Fève y Iover), para encargarle las Observaciones al Concordato de 1753 rue, con la caída del ministro quedaron inéditas. Tampueo se publicaron el Tretudo de /a Regalía ni las Reflexiones histónicas al nomisino Conourdeto de Campomanes.

Más afortunado fue Mayáns en sus relaciones intelectuales, espocialmente en su correspondencia con Andrés. Marcos Burriel, a quién animó en su evolurión hacia la historia crítica. Gencroso, celebró el nombramiento del jesuita como director de la Comisión de Archivos, animándole a publicar cuanto antes los doeumentos inéditos cuyo coneciniento, aseguraba, cambiaría la laz de los

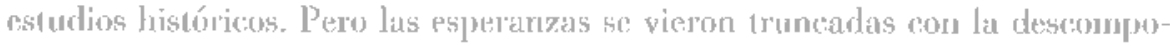
sición del equipo (árvajal-lingenada-Rávago y la subida al poded de Ricardo Wall, que anumciaba la extensión político-sescial de los manteistas con (iampomanes, quien (cn su testanlento) considerará el primer destino en la Asesoría de (orreos (en 1757) contu «el principio de mis servicios y atlelantamiento hasta llegas al ampleo de Gobernador del Gonsejos ${ }^{1:}$.

Fu conlaste con esas dificultades encontradas en Fsingaña, Mayáns es acogirlo con anabilidad en al cxuranjero. Uno de los miembros de la familia Deville puso en relación al valenciano con Gerardo Mrerman, jurista y polílico

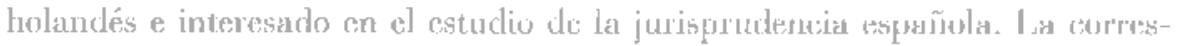
pondencia fue lecmeda. Colaboraron en la edición de Novus thesamus intis civilis et comonici, que veníc a completar el Thesaurus larts Romani de Fverharr (oto. Mayáus envió libros españoles a Holanela, dando a conocer nuestro pasado literario e historiogeáfío, hasta facilitat el conosimiento de Feijoo y líórez. Recibió, en cambio, las nuevas ediciones ele juristas aumpens o less recientes trabajos sobre humanismo.

Ahora bien, Meerman se convirtió en el jofe re las relariomes culturales de Mayáns en Europá, además de difundir su obrá eu los Páíses Bajos, donde el valenciano pulslicós sus Disputationes luris (1752) y De bello rnistico valentino de: Miñana (1743).

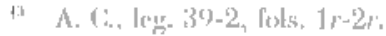


Una tupida red de conunicaciones hizo posible esta proyección. Meerman habló de la hiblioteca mayansiana a David Clement, que publicaba una serie de volúnenes en su serie Bibliothèque currense et critique. Clement solicitó un catálogo de la biblioteca mayansiana, pero la empresa era de tal calibre que se: limitó a los libros de granática y retórica, como puede verse en specimen bibliothecae hisprno-maiansiance (1753). La colaboración, anuque contimuó, no quedó plasmada en nuevas publicaciones, debido a las consecuencias de la Guerra de los Siere $\Lambda$ ños y a la muerte de Clement.

Similar fue el caso de la Acardenia Latina de Iena. De nuevo Meesman hizo de impermediario y los acarlémicos nombram socio de la doca institución al valenciano. liste redactó el preceprivo trabajo, Tractatus de hispenu progenue vocis 67 , pue en doble copia fue enviado a Jena por los enbajadores del lemperio (Migazzi) y de Inglatera (Kecne). También la guerra impidió su edición, que sólo en 1779) apareció en Madrid en la imprenta de Sancha. Queda, sin cmbargo, la correspondencia latina de Mayáns con el director de la Acarlemia, Joliamn E. I. Walch en que el propio Maýús exponía sus criteros, acusadamente críticos, sobre la epigrafía latina comservada en lispaña. Además, el mismo Mecrman puso al valenciano en redación con Srodtunam, que había publicado Ceschichte des edlen Herm Gregorms von Mayáns und Siscar (1740). La alegría de Strodtman fue inmensa y su soliciturl de unos apuntes biográlicos provocó la redaceión por palte de Mayáns de G. Muiunsii, generosi valentini, Vite, aparecirla en Wolfenbutel (1750) a nombre del mismo Strodiman, rector de Gimmasio de Osnabrük.

Lás relaciones de Mayáns con les alcmanes sem una prucba de interés por la cultura espanola entre los hombres de levas del mundo germánico. Parecían tener un especial interes en pasar a sus amigos la antoreha de la curiosidarl por mussira historia a través de Mayéns. De Merman a cilement; de Cilement a Carlos (: Pluer, agregado cultural de la embajada danesa en Madrid; de Pluer a la universirlad de Corjnga. Fstas cimenstancias explican la fama del erudito ente los intelectuales alesnanes, fue encontraron un inferlocutor competente y atento en sas respuestas llenas de comprensión y buen sentido.

No podemos dejar de aludir a las relaciones de Mayáns con los Ciramer, libreses de Ginebra, también propiciadas por Merman. La importancia de esta comunicación radica en que Cramer se convirtió en uno de los abastecedores de los libros más significativos del movimiento ilustrado europeos. Él editor ginchrino puso en contacto a Mayáns con Voltaire cuyas obras le enviós le commicó inmediatamente la aparición de la Enciclopedia y, a solicitud del solitario de Oliva, Sue remitiendo Montesquieu, Burlamaqui, y Pufendonf. 
Gramer, que visitó Oliva, se convirtió en un interlocutor de la ilustración racionalista con el cnvío de libros que, pese a las trabas inquisitoriales, llegaban a manos de los españoles interesados.

Data su formación jurílica y humanística, así como sus preocupaciones filosóficas, Mayáus tenía que conocer por necesidad las corrientes racionalistas más o nemos radicales. Y desde el campo del Derceho lo llegaron las prineras manilestaciones del racionalismo enropeo. Este primer súmoma resultri visilole en Epistolurum libsi sex (1732). Enore los móliphles jurisias cilados (humanisuas, católicos y protestantes) hay quo aludir a Grocio por su carácter simbólico.

En un jurista de: prestigio pareciáa necesario el conocimiento de I-lugo

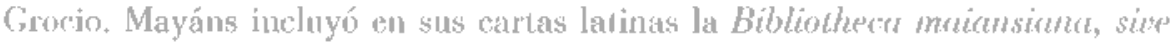

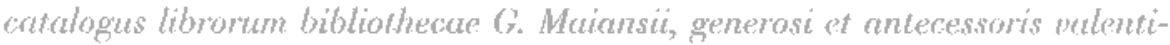
ni, libri invidici. En esto calálogo cita los libros juridicos que posec del loolandes: De mat libese, De mathos (10:33), y emite su juicio: «Dignissmi sum

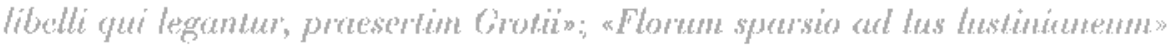

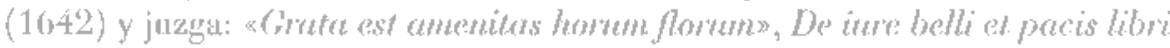
bes $(1650)$, con su juicio: "Magna est hatus operts doctrine, maior enditio".

Cinno puede observarse su juicio fuc esurictamente técnico, independiente de su calificavión religiosa. Y on esta línea mantiene su criterio en el prólogo: alaba su erudición, colelyra sus datos pertenecientes a la listoria des su timpo así como la aportación al progreso de las ciencias, aurque, confiesa con claridad, no siempre mantiene la misma belleza de estilo.

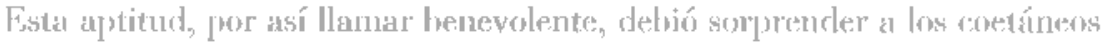
a juzgar por las palabras de Burriol, que créa conveniente que d valenetiano hubiera nobado el carácter religioso de Gocio: "No sé qué resultará sobre la condenación de Crocio a quier Vd. no porte tacha algumen en indice de su

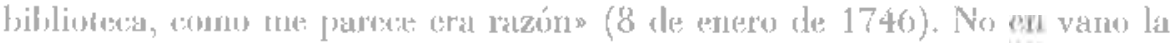
Inquisición anabó incluyendo las obras del holandés en al índice de libros prohilbides. Para que, sa: vea col ambiente y pueda juzgarse con exactitud las anteriores palabras de Mayáns, hay un texto de Burriel, en carta al valeneiano, que expresa el ambiente cultural del monernto: a Como el P. Ciasani está entargatalo de la impresión nueva del Expurgatorio, quicre que se prohíban in totum la sobras de Hugo Crocio de todos los géneros, pouque dice que es el fautor de el atuísino práctice y indiferencia en materia de religión que tanto reina en el Norte y que así le citan como su orácalo varios libros que por esto se han prohibido. Yo le he instado a due se tomase un temperamento an esto pues, aunque 
antes es la religión que nada, pero que podrían expurgarse con rigor y dejar lo demás. Instrúuame Vind. en esto y especialmente de sus libros «De iure belli et pacis» y de los demás que sea de Jurisprudencia» (20 de noviembre de 1745).

Gocio era, por tanto, autor peligroso. En 1750, en su censura a la Oratio ad Divinam Srapientiam, ponmeiada por Lorenzo Boturini en la Academia Valenciana, expuso cou claridad su pensaniento sobre cl derecho natural y de gentes. Botumi partía del criterio de Vico, con su eensura de los principios de Grocio, Seldeno, Pufendorf y Hobles. En esta límea tenía que ser aceptado por Mayáns que establecía en Dios y la creación el origen del derecho natural. "A esto se reduce el verdadero derecho natural que, por ser propio de los hombres, se Hama de las gentes, coyo estudio es ma utilísima meditación de la Divina Providencia, suprema legisladora, contra el gravísimo error te Iugón Crocio. que se aucví a escribir tque aunque no hubiera Dios haloría algún derecho natural, como si puclicra haber algo sin Dios o efecto sin causa. En semujante crror incurrieron Tomas Hobbes y Benito Espinosa, no rueriendo conocer esia ley superior, antecedente a roda costumbere y parto, suponiendo una razón sin dirección, que es lo mismo que decir, una razóm irracional» ${ }^{14}$.

En 1781, Campomanes (siendo dúm primer fiscal) mandó hacer un catálogo de la bibliotera para su uso particular (3.913 ohras en 6.899 tomos), y el Derecho natural y de gentes estaba encabezado por Grocio y Pufendorf, mostrando ciesta preferencia por Hugo Grocio debido a su «mélodo maravilloso de redneir la Jurisprudencia a sus principios», a la vez que recurre al contratualismo grociano para defender las regalias frente a la Iglesia calólica.

Sin embargo, el fiscal Camponanes, en su, Jucio Imparcial cita lanto a Grocio como a Pufendorl en $1768^{15}$, autores que presenta en su non-nato plan de esturtios de la nueva universidad española, al igual que tampoco fructificaría el plan universitario de Mayáns, encargado por su amigo Manuel Roda, Socretario de (iracia y Justicia (deste 170.5), wras ser nonubrato el erudito valenciano Alealde de Ciasa y Corte con una pensión vitalicia (en su viaje a Madrid en octubre-diciembre de 1766): entonces conoció personalmente al fiscal asturiano, conno ya se indicó anteriormente. (Mayáns fallecería el 21 de diciembre de 1781).

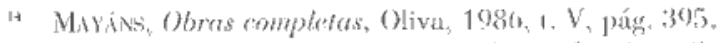

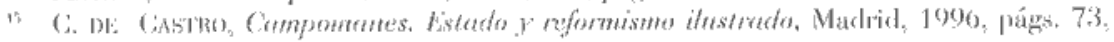

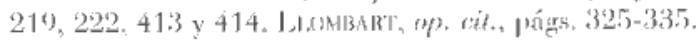


Sobre el conocimiento de Mayáns acerca de Suárez nos lo avala su carta a José Nebot (Oliva, 7 diciembre 1750): "Que el Concilio Lateranense V nes estaba admitido en España puede verlo en Suárez, tomo V, en $3^{\text {: }}$ parte, disputación 23, artículo 7, número 1». Más dudas tenemos en torno a su profundización en la doctrina suareciana, ya que en otra carta de Mayáns a I. B. Herman (Valencia, 6 de enero 1770) ante la descripción del parido antitomista, me las «teoríss de Suritzz con el molinismo, las dostrinas probabilistas o el regicidio ${ }^{\text {th }}$.

En manto a Campomazes, salvo Bustos que cita a Suáréz tras Molinal?, sólo conocemos a su liógrafu Concepción de Castro ${ }^{1 \%}$ que lo nombra con los jesuitas postridentinos entre Belarmino y Mariana y, en otro lugar, después de Molina como propia escnela teológica y moral; adenás de no aparcerer obra alguna de Suárcz en la citada biblioteca particular que el propio fiscal asturiano ordenó catallogar en $1781^{19}$. Siendo tal fiscal asturiano el impulsor de la real célula de la extinetón de la «escuela jesuítica", tras su dictanen (\$1 diciembre $1766)^{20}$ (on borradores sohre cl motín de Esquilaphe", detomantes de la expulsión de los jesuitas de lispaña (expulsión prevista por Mayáns con antelación, el 2 de fobrero y el 2 de abril de 1767$)^{2}$, no sorprende que el erudito valenciano y el fiscal asturiano descuidaran la doctrina de Suález, al igual que la Ilustración en general.

Según lierrater Mora, el baróu sueco (desde 1694) c hijo de un pastor protestante Samud lieiher von Pufendorf (16,32-1694), habúa sido influenciado por cl también frotestante. Hugo de Groot o Ifugo Crocio (158:31645). Este, nacido en la cindad holandesa I)elft (donte sería enterado), se introdujo en el Derecho en la universidard de Leyden, y en París desto 1594. Historiógrafo de los Estados Generales de los Países Bajos, y fiscal de la Corte de I Iolanda (a parlir de 1602); consejero pensionado de Rotterdan en $1613 \mathrm{y}$,

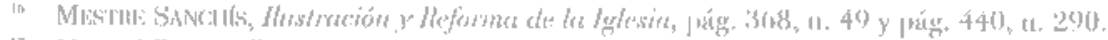

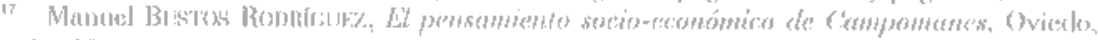
1982 , pág. 28.

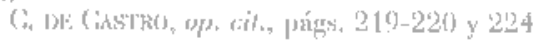

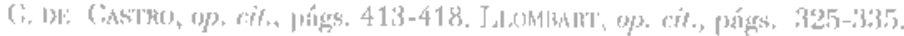

A. (i., Jeg. $45-4$.

A. 1 ... leg. $41-14$.

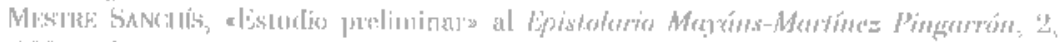
Valpucia, 1088, prígs. 3, 1:3, y 60). 
dos años después, embajador en Londres; al volver a Holanda, intervino eu las guerras de religión hasta ser cucarcelado en Loewenstein, de donde escapó en 1021 a Francia, cuyo rey, Luis XIII (1610-1643, hijo del borbón Enrique IV. rey de Navarra en 1562 y de Francia en 1589) le concedió asilo político y empleo oficial; en su retomo a Holanda (1625) vuclve a huir a llamburgo, donde el canciller conde $A x e l$ de Oxentiern (1583-1654, consejero del rey sueco Custavo Adollo (1611-16.32) y, tutor de Cristina reina de Suecia, tras la muerte de su padre en 1632 a su abdicación en 1654 al hacerse católica) le nombró cmbajador, permaneciendo en Estocolmo hasta que dicha reina Ciristina le designó su representante en París, pero dimitió en 1645 y marchó a Rostock, muriendo el 28 de agosto del mismo 1645.

En cambio, Pufendorf, nacido en Chemnitz (Sajonia), después de sus estudios de Teología y Derecho en Leipzig (16.54), y Filosofía y Matemáticas en Jena (1657), trabajó en la embajada de Suecia en Copenhague (1659) y en la Haya $(1000)$ hasta ser el inaugurador de la primera cátedra de Derecho natural y de gentes en la universidad de Heidellerg, y deste 1672 en la de Lund (Suecia), si bien (en 1086) se dedicó a la investigación y a la política como historiador y consejero de Estado en Suecia, y luego en Alemania al servicio del clector de Brandeburgo (en 1680), regresando a Suecia para recilir el título nobiliario de barón, pero murio en Berlín (26 octubre 1694$)^{2 !}$.

Si Mayáns, Camponanes y la mayor parte de la llustración curopea conocía la obra escrita de Crocio (sobre filología, teología, filosolía y derecho como De Mari libero, De iure praedae, De iure belli ac pacis, etc.), (rocio 110 negó las fuentes de sus obras" a Vidi et speciales libros de belli iure, partim a theologicis scriptis, ut a Fancisen Vitoria». Y citón 26 veces a Váacjuez de Menchaca, 44 a Vitoria, 21 a Molina, y 3 a Suárez, pero su influjo total es de Suárez, según nuestra anterior aportución ${ }^{23}$, siendo interpretado (primordialmente por Brown Scot1) el silencio solne Francisco Suárez (1548-1617) acerca De legibus ante el anbiente terriblemente hostil desde la Defensio fidei (en 1612) por parte de las cortes de París y Londres, influyendo solore el diplomático Grocio.

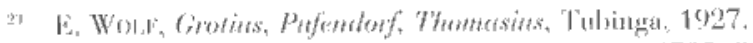

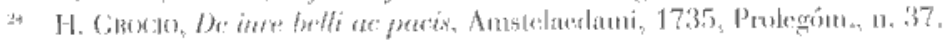

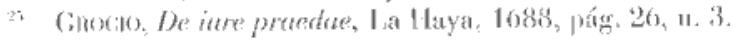

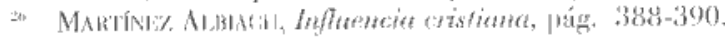


Así pues, en la moralidad del acto recurre a Dios, autor de la naturaleza ${ }^{27}$ : "Ins naturale est dictatum rectae rationis, indicans alicui actui, ex eus convenientia aut disconuenientia cum ipsa natura rational, inaesse moralem turpitudinem aut necessitatem moralem ac consequenter ab auctore naturae. Deo, talem actum netari aut praecipi».

Y menciona «las centellas de la luz divina» en su De iure praedae ${ }^{2 *}$ : $E s t$ quidem ista ratio nostro vitio obnobilata plurimum, non ita tamen quia conspicua restent semina divinae legis, quae in consensu gentium maxime, apparent (...). Placuit autem plerisque hunc ip sum consensum ius naturae secundarum, seu ius gentium primarium appellare».

Mas a este escrito del invierno de 1604 añadió la «nova declaratio», donde introduce e] pensamiento de Suárez en su De legibus ${ }^{20}$, y a partir de 1625 la afinidad es inmensa al comparat ambos textos:

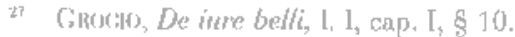

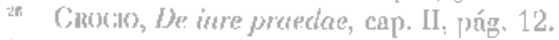

(2) Francisco Surkliz, Opera numica. De legibus, París, Vives, 1856, II, XIX, 8, 5, 168-160. 
"Non ea praecipit ant vetat. quae per se ant suapte natura debila sumt aut illicita: prapcipiondo debita farits.

"Ius gentium non ut ius naturale, ex certis rationibus oritur; sed e.e voluntate gentium (...). Reeta illatio, ex natura principuis procedens, ins thaturale indical; commumis aliquis consensus, ins gentiums.

"Hoc autem nom est iass illud gentium proprie dictum (..) ac proinde ins gentium impropre dictum introducerelar".

"Neque enim pertinent ad muluam gentium inter se societal.em».

"Lsse ius civile plurintam gentium distributim».

"lhde $a b$ uno poputo, alies incomsultis mutari potnit (...). Onod a singulis (gentibus) tolli potest).

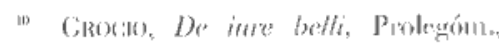
11.40; I, X, ; II, III, 5; II, VIII, 1; II, XV1, 2; XVJII, IV, 1-2,
"Itaque nom prohibet mala quin mala sunt, sed prohibendo facit males.

"Differt autem primo ac pretectpue ius gentium a naturali quia (...) non infert necessitatem rei praeceptae ex sola rei natura per evidentem illatione'm ex principits naturalibus; quict quidquid huinsmodi est, noturale esto.

"Prior modus videtur mihi proprissime continere ius gentium; re ipsa distinctum a iure rivili (...). Si sermo sil de proprto iure gentiums.

"Neque enim habent veluti pro fine proximo conmunicationem societatem et communicationem omnium nationum inter sem.

"Uth est in illa republica, est tantum civile intrinsece (...). Proxime videnur spectrore potius ad ins civile».

«Potest immutari a petrticulurt regno nel republica (...); potest ab una in se mutari, licet aliae uon consentiant".

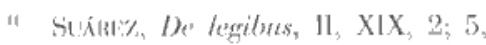
$106 ., \mathrm{XIX}, 8 ; 5,168,11, \mathrm{XIX}, 10 ; 5,109-170$. II, XX, 1-10; 5, 170-172. 
Siguicndo a Suárez, para Grocio así como el derecho intemo ha nacido necesariamente para procurar la utilidad de los ciudadanos de un país, el bienestar de ese gran conjunto ("magnae illius muiversitatiss) de ciudades exige un derecho común a todas; y el fumbamento de esa gran comunidad que abarca a todo el género humano, o a la mayoría de los pueblos (xeommentitas quae genus hamanum aut populos complures inter se colligato), descansa en la múltiple inverdependencia que los liga y enlaza, y exige consiguientemente un derecho que la regule y ordene.

Grovio remió, por vez primera, tonas las cuestiones relativas al derecho de la guerra y de la paz por los Estados tn un sistema organizado y coherente. Aunque Francisco de Vitoria fuera el fundador del derecho unter gentes o internacional, Grocio lo trató de un modo seuarado, como una disciplina independiente, dando a la comanidad internacional ana organización sistenática. Además, sc apoya cu la necesidad del respeto al soberano, pues supone que éste lia sido clevado al poder por consentimiento tácito de los individuos, y que éstos mecesitan de arpuél para namiturer la fuerza legal de sus relaciones entre sí, pero con viturios absolutistas ${ }^{41}$.

Sin embargo, el barón Pufendorl inició el proceso antiteológico de la Jurisprudencia, consumando el divorcio erutre el Derecho y la Moral: asigna a acuél el fuero externo, y a ésta al interno ${ }^{34}$.

Insislimos en que Máyáns, Ciamponanes y, al menos, la mayor parte de la Ilustración europea descuidaron las l'uentes de Grocio por los mismos motivos que el diplontático holandés ante el ambierıte social antijesuítico en Londres. París y Madrid. Pero Mayáns y Camponanes, al igual que los regalistas espanoles (incluyendo a los jesuitas: Févre, confesor de Felipe V, y considerado como «it nostro mortal enemico» al cuiticarlo el nuncio español ante Roma, el 31 de agosto de $1745^{\circ}$, hasta su alejaniento del trono con el cese de Rávago en 175.5) $)^{\text {th }}$, defendicron, especialmonte el asturiano, el fortalecinicnto del poder rlel monarca como único indiscutible: Cimpomanes, como expervo aho-

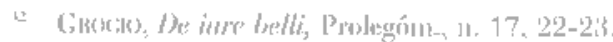

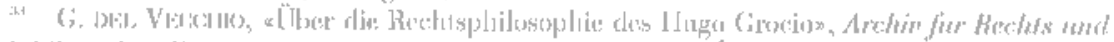

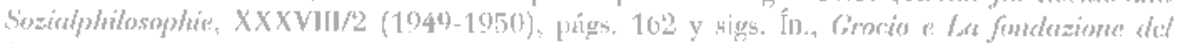

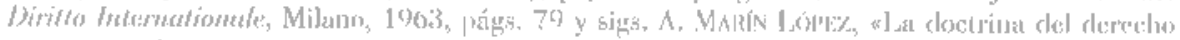

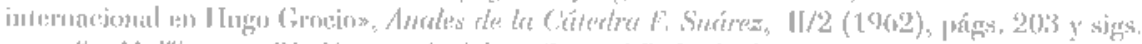

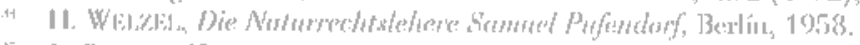

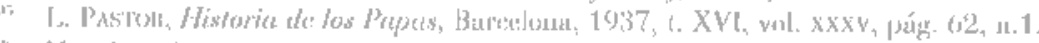

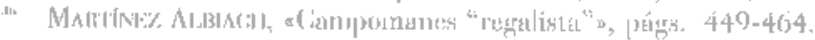


gado y hábil político, fundamentaba, en cada caso concreto, alegando sus argumentos, tomados de diversos cuerpos teóricos, relativos al poder político, para argumentar el derecho del príncipe secular a resistir y defenderse contra el Romano Pontífice si éste se entrometía en lo que manifiestamente fuera de jurisdicción temporal, no espiritual, sin importarle aceptar el contractualismo de Grocio para delender las regalías del soberano ${ }^{37}$, e incluso admitir el divorcio de Pufendorf entre Derecho y Moral. De ahí la preferencia por el barón e hijo de pastor protestante sobre la implicita dependencia de Crocio ante Suárez, facilitando el regalismo hispano de linales del siglo xyıll, al que no llegó Mayáns por su profunda religiosidad ${ }^{3}$, expuesta en el manuscrito publicado por Mestre en 1995: Gregorio Mayáns falleció en 1781, como manifestamos anterionente.

La preferencia por Pufendorl, de parte de los ilustrados españoles, primordialmente de Camponanes, gira en torno al ideal de la iglesia nacional para confirmar las regalías de la corona", según confirman sus biógrafos Coronas y Llombar1" : aspiraba, como los demás ilustrados hispanos, a una iglesia nacional, donde cupiera un mayor papel a los laicos y que estuvicra además dirigida por los obispos españoles, según el modelo de los primeros tiempos cristianos, y con capacidad del poder civil para intervenir, pues los eclesiásticos «son ciudadanos, miembros de la república y sujetos, por tanto, en todo lo que no sea puramente espirilual, a la potestad del soberano». Éste tamporo puede remunciar a la intervención, pues su regalía no es sólo un derecher, sino una obligación; sin negar el primado papal, no acepta las extralimilaciones de la curia romana, para poder combatir el «poder indirecto» ${ }^{4}$.

Por tanto, la doctrina de Pufendorf, pese a apoyarse en Crocio (y con recurso die éste a buárez), encaja mejor con los ilustrados en la segunda nitad de nuestro siglo XvII, realidad histórico-social que ilumina los siglos XIX y Xx de España, gracias a las aportaciones de Mayáns y Campomanes.

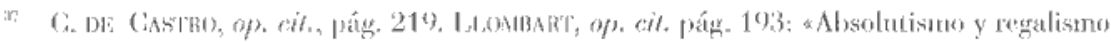

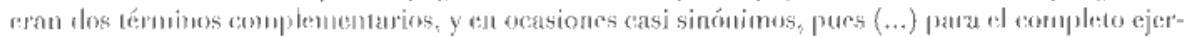

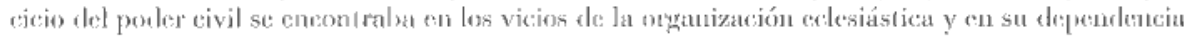
de un priseipe cxtranjersos.

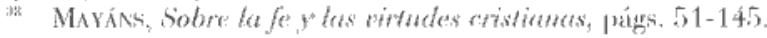

3" Vick. wata 5 .

4li firl. nola 12 .

\# C. ar (iAstro, op. cil., págs. 220-220. 